\title{
Effects of Different Extraction Methods on the Properties of Pine Cone Polysaccharides from Pinus koraiensis
}

\author{
Qiang Zhao, ${ }^{\mathrm{a}}$ Jie Qin, ${ }^{\mathrm{b}}$ Hairong Wang, ${ }^{\mathrm{a}}$ Jingyu Wang, ${ }^{\mathrm{a}}$ and Xueming Zhang ${ }^{\mathrm{a}, *}$ \\ In order to realize the resource utilization of the Pinus koraiensis pine cone, \\ the polysaccharides were obtained by a traditional hot water extraction \\ process, and the optimal extraction process was confirmed. The effects of \\ a traditional hot water, alkali, and ultrasound assisted extraction on the \\ yield and properties of polysaccharides were studied. The structure, \\ monosaccharide composition, and content of polysaccharides obtained by \\ different extraction methods were analyzed by means of Fourier infrared \\ chromatography and liquid chromatography. Effects of polysaccharides \\ extracted by different methods on the antimicrobial activity of \\ Staphylococcus aureus and Escherichia coli were studied. The \\ scavenging effect of DPPH free radicals were also examined. The \\ optimum condition of the hot water extraction was identified as an \\ extraction temperature of $100^{\circ} \mathrm{C}$, a ratio of material to water at $1: 16(\mathrm{w} / \mathrm{v})$, \\ an extraction time of $4 \mathrm{~h}$, and a yield of polysaccharide at $10.2 \%$. In \\ comparison, the yield of the pine cone polysaccharide was improved \\ significantly to $29.1 \%$ by using $\mathrm{NaOH}$ solution extraction method. The \\ FTIR spectra of polysaccharides showed that the samples possessed the \\ typical chemical structure of a polysaccharide.
}

Keywords: Pine cone; Pinus koraiensis; Polysaccharide; Extraction

Contact information: a: College of Materials Science and Technology, Beijing Forestry University, 100083, Peoples Republic of China; $b$ : Dalian Institute of Chemical Physics, Chinese Academy of Sciences;

*Corresponding authors: zhaoqiang@bjfu.edu.cn;xm_zhang@bjfu.edu.cn

\section{INTRODUCTION}

Pine cones are found on gymnosperms, such as Korean pine, Chinese pine, Pinus armandi, and Pinus yunnanensis (Lv et al. 2010). Pine cones contain a variety of chemical components including terpenoids, polysaccharides, polyphenols, brass, and fatty acids. Among these, pine cone polysaccharides mainly consist of ribose, rhamnose, arabinose, xylose, mannose, glucose, and galactose in different molar ratios (Xu et al. 2012). Pine cone polysaccharides have a strong reducing ability and good scavenging effects on various free radicals. It has been shown that polysaccharide fraction isolated from pine cones of Pinus parviflora Sieb. \& Zucc. exhibited various pharmacological activities, such as antimicrobial, anti-HIV, and stimulation of tumor necrosis factor (TNF) production (Sakagami et al. 2010). Moreover, with the increase of polysaccharide concentration in a certain concentration range, the scavenging ability is continuously enhanced (Wang et al. 2015; Zhang et al. 2015). In addition, pine cone polysaccharides have immune regulation effects (Moradali et al. 2007; Zhang et al. 2010, 2014; Xu et al. 2012), and anti-tumor effects have also been linked to pine cone polysaccharides (Sakagami et al. 1987; Tang et al. 2003; Lu et al. 2008; Lin et al. 2013). 
Treatment with acidic polysaccharides from hot water extracts of Japanese pine cones resulted in a significant prolongation of survival time in ascites mice (Sakagami et al. 1987; Lv et al. 2010). The polysaccharide extracted from Japanese pine needles has anti-tumor, anti-bacterial, and immunological activities (Tang et al. 2003). PC6 and PC7 from the same polysaccharide in Japanese pine inhibit the replication of HIV in vitro (Tang et al. 2003). Lv et al. (2008) found anticancer activity in pine cone and pine nut shell by inoculating mice with S180, U14, and Hep A. The results showed that pine cone had a better anticancer effect. Apart from polysaccharides in pine cone, some volatile components also have some biological activities such as antiviral and antibacterial (Lv et al. 2010). The anti-tumor component of pinecone extract also has the function of protecting influenza virus. Acid PCE can significantly inhibit the growth of influenza virus in $\mathrm{Ma} \mathrm{Da}$ dog renal cell line. It can significantly inhibit the synthesis of virus protein in infected cells (Lv et al. 2010). Moreover, it has been reported that pine bark extract has an antiproliferation effect on human breast cancer cells, and shows strong DPPH radical scavenging activity, reducing power, nitric-oxide scavenging activity and ferrous-ion chelating ability (Yu et al. 2008).

Approximately half of the world's Korean pine resources are distributed in the area from Changbai Mountain to Xiaoxing'anling in Northeast China, which provide sufficient raw material to develop high value utilization of pine cone (Wang et al. 2013; Zhang et al. 2016). At present, a large number of pine cones are only burned as cheap fuel, causing a great waste of resources. In this study, the extraction process of pine polysaccharide was optimized, and the monosaccharide composition was analyzed. The antibacterial and antioxidative properties were studied to provide a theoretical basis for the high value utilization of Pinus koraiensis pine cone.

\section{EXPERIMENTAL}

\section{Materials}

The pine cone material used in the experiment was collected from the Pinus koraiensis species in November in the northeast of China.

\section{Preparation of Pine Cone Raw Materials}

After the pine seed of the Pinus koraiensis raw material was manually removed, it was oven-dried at $50{ }^{\circ} \mathrm{C}$, and the dried pine cone was pulverized into a powder and sieved with a 50 mesh sieve to ensure uniformity of the raw material. Next, $100 \mathrm{~g}$ of the sieved raw material was mixed with four volumes of ethanol, and the mixture was extracted in a micro-boiling state for $2 \mathrm{~h}$ with a Soxhlet extractor. The alcohol extract was filtered, and the extraction was repeated twice.

\section{Preparation of Pine Cone Crude Polysaccharide}

Optimization of process conditions and polysaccharide extraction by traditional extraction methods

The polysaccharide fractions were successfully isolated from pine cones of Pinus koraiensis by water extraction and ethanol precipitation (Xu et al. 2012). In brief, a total of $6 \mathrm{~g}$ of pine cones were extracted using distilled water according to the ratio of material to liquid designed by the orthogonal test. After the water extraction treatment, the residue was removed by centrifugation and filtration. The pine cone polysaccharide extractives 
were concentrated by evaporation to approximately $30 \mathrm{~mL}$, and then precipitated by adding $300 \mathrm{~mL}$ of ethanol. The obtained product was centrifuged to remove the supernatant, and the precipitate was dried. The extraction was carried out under optimal conditions, and the obtained sample was named as sample A.

\section{Extraction of pine cone polysaccharide with $\mathrm{NaOH}$}

The pine cone was extracted with $1 \% \mathrm{NaOH}$ instead of hot water under the above optimal conditions. The obtained polysaccharide sample was named as sample B.

\section{Ultrasonic assisted polysaccharide extraction process}

The process parameters of ultrasound assisted extraction of polysaccharides from Pinus koraiensis were as follows: material-to-liquid ratio of $1: 30$, temperature $60{ }^{\circ} \mathrm{C}$, time $20 \mathrm{~min}$, and ultrasonic power $210 \mathrm{~W}$. The experimental group (C) was obtained with ultrasound assisted extraction, while the control group (D) was also prepared with the same conditions without ultrasound assistance.

\section{Preparation of Purified Pine Cone Polysaccharide}

Approximately $1 \mathrm{~g}$ of crude polysaccharide was put in $100 \mathrm{~mL}$ beakers, and $20 \mathrm{~mL}$ of distilled water and $20 \mathrm{~mL}$ of $9 \%$ trichloroacetic acid were added. The mixture was stirred for 30 minutes to remove protein from the raw materials. The precipitate was removed, and an equal volume of ethanol was added to the supernatant to stir overnight. The supernatant was removed by centrifugation, whereupon $6 \%$ hydrogen peroxide and $3 \% \mathrm{NaOH}$ were separately added to the precipitate. The mixture was sealed at $80{ }^{\circ} \mathrm{C}$ for $1 \mathrm{~h}$ and vented at appropriate times to remove the pigment therein. After the reaction, an equal volume of ethanol was added and stirred overnight, and the supernatant was removed by centrifugation. After the ethanol was evaporated, the purified polysaccharide sample was obtained by freeze-drying. The purified samples obtained from crude polysaccharide of $A$, $\mathrm{B}, \mathrm{C}$ and $\mathrm{D}$ were named as sample $\mathrm{A}^{\prime}, \mathrm{B}^{\prime}, \mathrm{E}$ and $\mathrm{F}$, respectively.

\section{Testing and Characterization}

\section{Infrared spectrum of the pine cone polysaccharide}

FT-IR spectra of pine cone polysaccharide samples were obtained on an FT-IR spectrophotometer (Tensor II, Bruker, Karlsruhe, Germany) using a KBr disk containing $1 \%$ finely ground samples.

\section{Determination of monosaccharide composition of the pine cone polysaccharide}

The monosaccharide composition of the purified pine polysaccharide samples A', B', $\mathrm{E}$, and $\mathrm{F}$ were analyzed by high-performance anion-exchange chromatography (HPAEC) system (Dionex ICS5000, USA) with pulsed amperometric detector and an ion exchange Carbopac PA-1 column $(4 \times 250 \mathrm{~mm})$. In brief, the purified pine polysaccharide samples were determined by hydrolyzing $\sim 5 \mathrm{mg}$ samples using $1.475 \mathrm{~mL}$ of $6.1 \% \mathrm{H}_{2} \mathrm{SO}_{4}$ for $2.5 \mathrm{~h}$ at $105{ }^{\circ} \mathrm{C}$ with occasional vibration. The hydrolyzate was diluted and injected into the HPAEC system. Six monosaccharides such as galactose, arabinose, mannose, rhamnose, glucose, and xylose were used as standards.

\section{Antioxidant detection of the pine cone polysaccharide}

To evaluate the free radical scavenging properties of pine cone polysaccharide, their reactivity towards a stable free radical, 2,2'-diphenyl-1-picrylhydrazyl radical (DPPH), 
was evaluated according to the literature with some modifications (Spizzirri et al, 2009) For this purpose, crude polysaccharides and purified polysaccharides $(10 \mathrm{mg} / \mathrm{mL})$ were prepared. Then, $0.1 \mathrm{~mL}$ of polysaccharides solution and $3.9 \mathrm{~mL}$ ethanol solution of DPPH $(0.025 \mathrm{mg} / \mathrm{mL})$ were mixed incubated in a water bath at $25{ }^{\circ} \mathrm{C}$ and, after $24 \mathrm{~h}$, the absorbance of the remaining DPPH was determined at $517 \mathrm{~nm}$. The scavenging activity of the tested conjugates was measured as the decrease in absorbance of the DPPH.

Antibacterial test of the pine polysaccharide and alcohol extractives

The antimicrobial activity of the prepared films against Staphylococcus aureus or Escherichia coli was qualitatively evaluated by the disk inhibition zone assay according to previously published paper (Pelissari et al. 2009).

\section{RESULTS AND DISCUSSION}

\section{Process Optimization of Pine Cone Polysaccharide}

As shown in Table 1, the three factors of the ratio of material to liquid, extraction temperature, and extraction time have different effects on the extraction of polysaccharides. It can be judged from the size of the range $R$ in the table and the degree of influence that leaching temperature is greater than the material-liquid ratio, which is greater than the leaching time. The optimum technological conditions for polysaccharide extraction were as follows: the ratio of material to liquid of $1: 16$, the extraction temperature of $100{ }^{\circ} \mathrm{C}$, and the extraction time of $4 \mathrm{~h}$. Under these conditions, the yield of polysaccharides in the verification experiment was up to $10.2 \%$. The final polysaccharide obtained with the optimum conditions was named sample (A).

Table 1. Results of the Orthogonal Experiment on Hot Water Extraction

\begin{tabular}{|c|c|c|c|c|}
\hline \multirow{2}{*}{$\begin{array}{c}\text { Sample } \\
\text { Number }\end{array}$} & $\begin{array}{c}\text { Ratio of } \\
\text { Material to } \\
\text { Liquid }\end{array}$ & $\begin{array}{c}\text { Extraction } \\
\text { Temperature }\left({ }^{\circ} \mathrm{C}\right)\end{array}$ & $\begin{array}{c}\text { Extraction Time } \\
(\mathrm{h})\end{array}$ & $\begin{array}{c}\text { Polysaccharide } \\
\text { Yield }(\%)\end{array}$ \\
\hline 1 & $1: 8$ & 80 & 3 & 7.18 \\
\hline 2 & $1: 8$ & 90 & 4 & 8.42 \\
\hline 3 & $1: 8$ & 100 & 5 & 9.07 \\
\hline 4 & $1: 12$ & 80 & 4 & 7.54 \\
\hline 5 & $1: 12$ & 90 & 5 & 8.47 \\
\hline 6 & $1: 12$ & 100 & 3 & 9.32 \\
\hline 7 & $1: 16$ & 80 & 5 & 7.91 \\
\hline 8 & $1: 16$ & 90 & 3 & 8.64 \\
\hline 9 & $1: 16$ & 100 & 4 & 10.25 \\
\hline Mean value (k1) & 8.223 & 7.543 & 8.380 & \\
\hline Mean value (k2) & 8.443 & 8.510 & 8.490 & \\
\hline Mean value $(\mathrm{k} 3)$ & 8.687 & 9.300 & 8.483 & \\
\hline Range R & 0.464 & 1.757 & 0.110 & \\
\hline
\end{tabular}




\section{Extraction of the Polysaccharide from the Pine cone by Alkali Solution}

Based on the determined optimal extraction conditions of traditional water extraction and alcohol precipitation, the distilled water was changed to a $\mathrm{NaOH}$ solution with a mass concentration of $1 \%$, and the obtained polysaccharide (B) was as shown in Fig. 1. The final yield was $29.1 \%$, which improved the extraction rate of the pine cone polysaccharide. The alkali solution extraction was higher than the water extraction rate because the alkali solution can break the wall of the plant cells during extraction of the polysaccharide (Xiang 2004). Without destroying the structure of the polysaccharide, a higher alkali concentration results in a stronger wall-breaking effect. The alkali makes the polysaccharide more soluble in the alkali solution, and the use of alkali extraction increases the permeability of the polysaccharide so that the amount of the polysaccharide remaining in the pine cone is greatly reduced. As a result, a higher extraction rate was obtained.

\section{Ultrasound Assisted Extraction Experiment}

The process parameters of ultrasonic assisted extraction of polysaccharides from Pinus koraiensis were as follows: a material-to-liquid ratio of $1: 30$, temperature of $60{ }^{\circ} \mathrm{C}$, time of $20 \mathrm{~min}$, and ultrasonic power of $210 \mathrm{~W}$. The final yield was $8.24 \%$. Comparatively, the yield of the control group was only $6.86 \%$.

In the ultrasonic assisted method based on water extraction and alcohol precipitation, the chemical bond between the pine polysaccharide and the plant cell wall (intercellular substance) was destroyed by simple mechanical action. The bond breakage makes it easier to separate from other substances in the cell, thus greatly improving the extraction efficiency and ultimately shortening the extraction time. However, excessive sonication, such as too much power and too much time, may cause the structure of the polysaccharide to be damaged which adversely affects its biological activity.

\section{Purification of the Pine Cone Polysaccharide}

The crude polysaccharide was purified according to the above procedure, and the obtained purified polysaccharide is as shown in Fig. 1. The yield of the experimental group (E) was $8.52 \%$, and the yield of the control group (F) was $11.58 \%$.
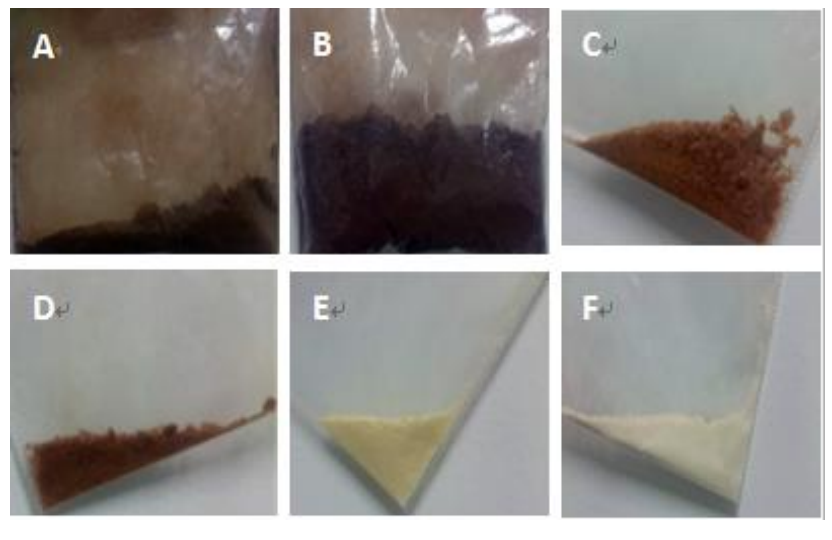

Fig. 1. Polysaccharide from pine cone of Pinus koraiensis obtained by different extraction methods and its purified polysaccharide. Note: A, obtained sample with traditional extraction process; $\mathrm{B}$, obtained sample with $\mathrm{NaOH}$ extraction process; $\mathrm{C}$, obtained sample with ultrasound extraction process; D: obtained sample without ultrasound extraction process (other treatment conditions were the same as C); E, the purified sample C; F, the purified sample D 
The yield of the experimental group obtained was lower than that of the control group. It was preliminarily believed that during the ultrasonic assisted extraction process, the ultrasonic wave caused damage to the structure of the pine cone polysaccharide, causing it to be lost during the purification process.

\section{Infrared Spectrum Analysis of the Pine Cone Polysaccharide}

Fourier transform infrared spectroscopy of Samples A and B

As shown in Fig. 2, the broad absorption peak around $3400 \mathrm{~cm}^{-1}$ was mainly a characteristic absorption peak caused by $\mathrm{O}-\mathrm{H}$ stretching vibration. A series of absorption peaks in the 2980 to $2800 \mathrm{~cm}^{-1}$ interval were characteristic absorption peaks due to the stretching vibration of saturated $\mathrm{C}-\mathrm{H}$. A series of absorption peaks in the 2360 to $2020 \mathrm{~cm}^{-}$ ${ }^{1}$ range are due to unsaturated $\mathrm{O}=\mathrm{C}=\mathrm{O}$, and characteristic absorption peaks caused by stretching vibration of $\mathrm{C} \equiv \mathrm{N}$ or $\mathrm{C} \equiv \mathrm{C}$. The absorption peak near $1600 \mathrm{~cm}^{-1}$ was caused by the absorption peak of the crystal water of the polysaccharide and the stretching vibration of $\mathrm{C}=\mathrm{O}$. The absorption band around $1400 \mathrm{~cm}^{-1}$ was produced by the symmetric stretching vibration of -COO-. The band at 1180 to $1000 \mathrm{~cm}^{-1}$ was produced by the stretching vibration of the ether bond (C-O-C) on the sugar unit. The absorption peak near $838 \mathrm{~cm}^{-1}$ was a characteristic absorption peak of the $\alpha$-glycosidic bond, indicating that $\alpha$-glycosidic bonds were present in the skeleton of the polysaccharide (Shen 2014).

The absorption peak intensity of the $\mathrm{C}=\mathrm{O}$ bond of $\mathrm{B}$ was stronger than $\mathrm{A}$, which indicated that the $\mathrm{C}=\mathrm{O}$ bond was increased in the molecule of the pine cone polysaccharide extracted by the dilute alkali solution. The introduction of metal ions had a certain promoting effect on the formation of carbonyl groups (Li 2013).

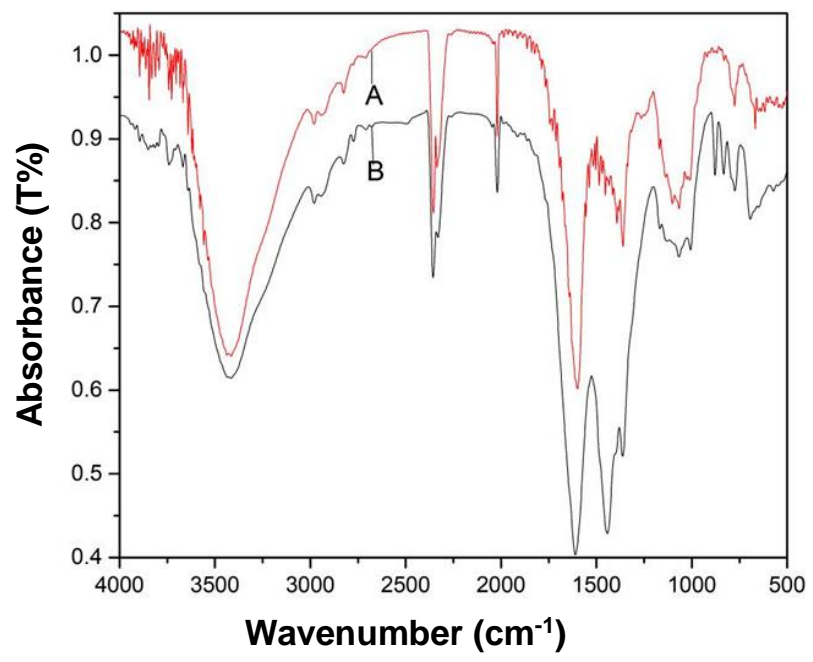

Fig. 2. Infrared spectra of samples A and B

Fourier transform infrared spectroscopy analysis of samples $C, D, E$, and $F$

The infrared absorption peaks of samples C, D, E, and F as shown in Fig. 3 were like the infrared absorption peaks of samples A and B. The intensity of the absorption peaks of the four samples in the range of 2360 to $2020 \mathrm{~cm}^{-1}$ was weaker or even less than that of samples A and B. The reason might lie in samples C, D, E, and F, which were all extracted at a temperature of $60{ }^{\circ} \mathrm{C}$, while samples $\mathrm{A}$ and $\mathrm{B}$ were both carried out at a temperature of $100{ }^{\circ} \mathrm{C}$. It is speculated that the substances in the A and B samples in the range of 2360 to $2020 \mathrm{~cm}^{-1}$ were organic impurities having a melting point higher than $60{ }^{\circ} \mathrm{C}$. 


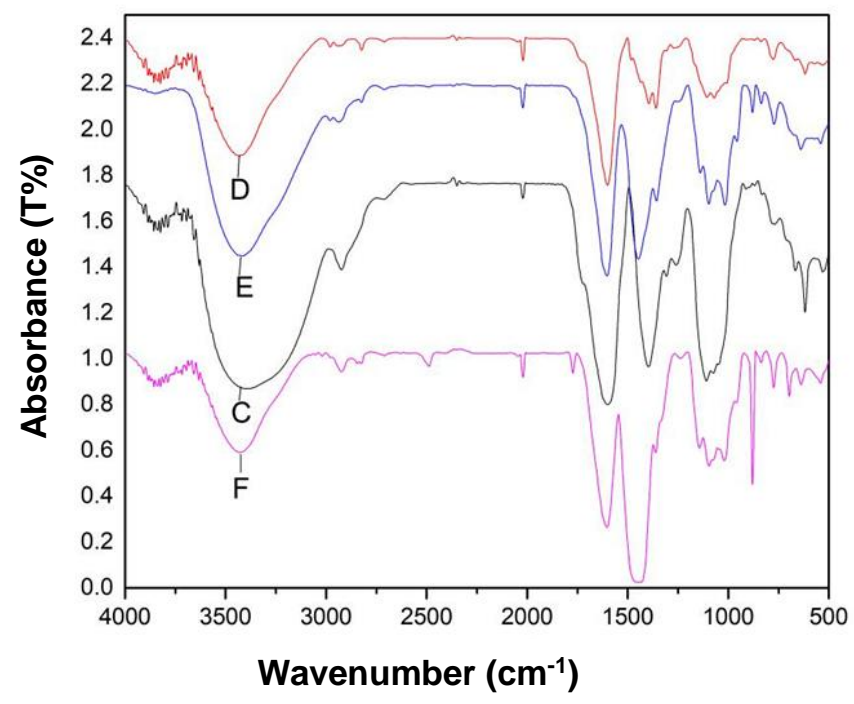

Fig. 3. Infrared spectra of samples $C, D, E$, and F

Analysis of Monosaccharide Composition of the Pine Cone Polysaccharide

The monosaccharide composition of four samples of Pinus koraiensis polysaccharides A`, B`, E, and F by liquid chromatography are shown in Table 2 . According to preliminary determination by liquid chromatography, the extracted polysaccharides of Pinus koraiensis contain four kinds of monosaccharides, which were glucose, galactose, arabinose, and mannose. The main monosaccharide composition of the four samples was galactose, followed by glucose and mannose, and the arabinose content was the lowest. In addition, it can be seen from the graph that the polysaccharide obtained by the lye extraction had the lowest mass of the monosaccharide per unit mass of the sample, which may be because of the monosaccharide instability in the alkali solution, and the isomerization and decomposition reaction occurring with the lye concentration increase (Li 2013). The mass of monosaccharide contained in the unit mass of $\mathrm{E}$ and $\mathrm{F}$ samples was also lower than that of A, which may be related to the low solubility of some polysaccharides with larger molecular weight at lower temperature.

Table 2. Monosaccharides and Constituents of Polysaccharides from Different Samples

\begin{tabular}{|c|c|c|c|c|}
\hline \multirow{2}{*}{ Monosaccharide } & \multicolumn{4}{|c|}{ Content $(\mathrm{mg} / \mathrm{mL})$} \\
\cline { 2 - 5 } & $\mathrm{A}^{\prime}$ & $\mathrm{B}^{\prime}$ & $\mathrm{E}$ & $\mathrm{F}$ \\
\hline Arabinose & 0.0246 & 0.0462 & 0.0168 & 0.0228 \\
\hline Galactose & 0.1026 & 0.3081 & 0.1310 & 0.1507 \\
\hline Glucose & 0.0415 & 0.1630 & 0.0687 & 0.0730 \\
\hline Mannose & 0.0239 & 0.0859 & 0.0245 & 0.0274 \\
\hline
\end{tabular}

Note: $A^{\prime}$, the purified sample from sample $A ; B^{\prime}$, the purified sample from sample $B$. $E$, the purified sample $C ; F$, the purified sample $D$.

\section{Antioxidant Analysis of the Pine Cone Polysaccharide}

According to the experimental results in Fig. 4, polysaccharide samples A, C, D, E, and $\mathrm{F}$ all had certain DPPH free radical scavenging effects. With the increase of 
polysaccharide concentration within a certain range, the DPPH free radical scavenging effect was continuously enhanced, and there was a significant effect of dosage. The scavenging rate of DPPH free radicals by water extracting polysaccharide A was much higher than that of other extraction methods. The scavenging rate of DPPH free radicals was similar for polysaccharide samples obtained by other extraction methods. At the same time, the unpurified polysaccharide was larger than the purified polysaccharide, and no ultrasound is added other than ultrasonic extraction.

The preliminary analysis showed that the trend was caused by the unpurified polysaccharide containing components such as proteins and pigments, and these substances may also contain some antioxidant components. The polysaccharides that had not been sonicated were relatively less damaged by the polysaccharide structure or other antioxidant active substances, and the antioxidant activity was more complete. Alkali extracted polysaccharide $\mathrm{B}$ had no scavenging effect on DPPH free radicals. The preliminary analysis was due to the excessive concentration of lye, the alkalinity was too strong, and the extraction time was too long, which led to the structure of the polysaccharide of pine cone and other active substances becoming severely damaged.

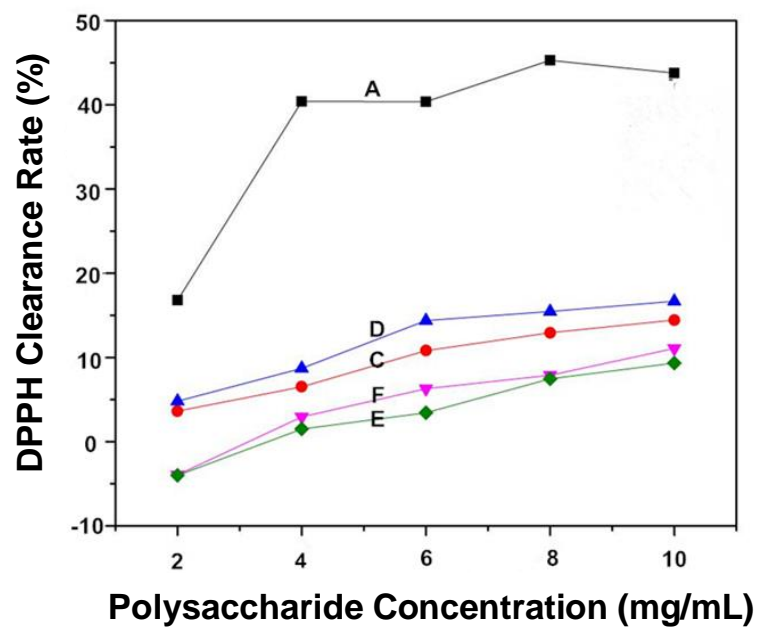

Fig. 4. DPPH clearance vs. concentration of different polysaccharide samples from the pine cone of Pinus koraiensis obtained by different extraction methods

\section{Antimicrobial Analysis of the Pine Cone Polysaccharides and Alcohol Extracts}

Antibacterial analysis of the pine cone polysaccharide

In the preliminary antibacterial test of six samples A, B, C, D, E, and F, no obvious inhibition zone was found in the respective medium. That is, no antibacterial activity was detected. This conclusion was different from other previous study conclusions (Meng 2010). After analysis, there are three main reasons that could account for these findings:

a. The amount of polysaccharide used in the detection process may have been too small, and the antibacterial property of the selected strain was not strong, resulting in no antibacterial effect.

b. The dissolution effect of the polysaccharide sample was not good, and it was difficult to fully contact the strain after inoculation into the solid medium containing the colony, so that the antibacterial effect cannot be applied to the bacteria in the medium. 
c. During the extraction process, the antibacterial property has been destroyed because of high temperature, lye or ultrasound.

\section{Antibacterial analysis of alcohol extracts from raw materials of pine cone}

The alcohol extract obtained from the pretreatment of the pine cone raw material was tested for antibacterial activity. There was an obvious antibacterial effect on E. coli. An obvious inhibition zone could be observed in the medium, as shown in Fig. 5, and the diameter of the inhibition zone was $3 \mathrm{~mm}$ measured with a Vernier caliper. No inhibition zone was observed for $S$. aureus.

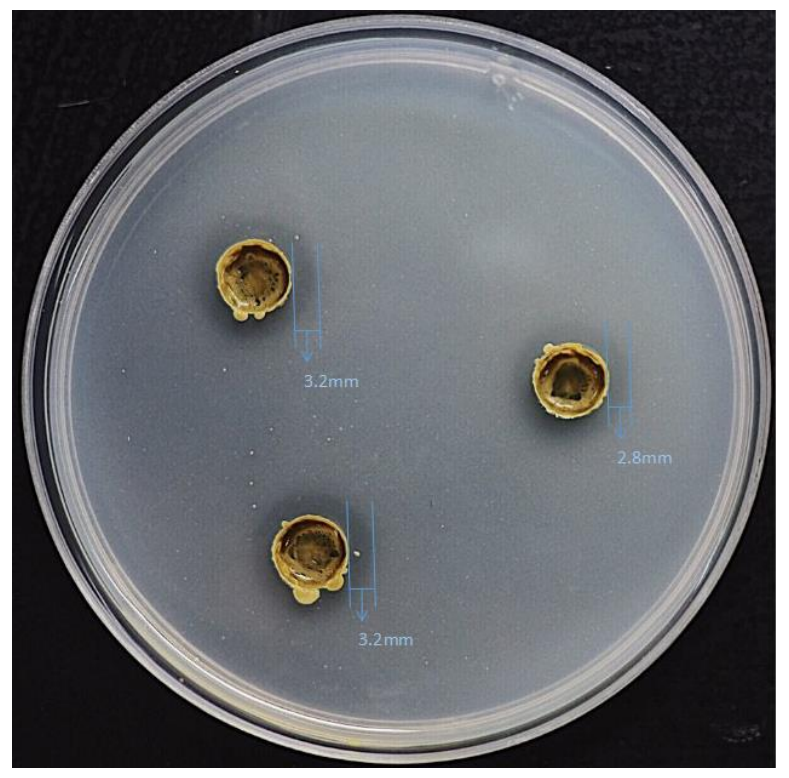

Fig. 5. Antibacterial effect of alcohol extracts of pine cone raw materials on $E$. coli

\section{CONCLUSIONS}

1. The polysaccharides from Pinus koraiensis were extracted by the hot water extraction method. The optimum extraction parameters were determined as follows: the ratio of material to liquid was $1: 16$, the extraction temperature was $100{ }^{\circ} \mathrm{C}$, and the extraction time was 4 hours. The yield of extracted polysaccharides could reach up to $10.25 \%$.

2. The monosaccharide components of polysaccharides extracted by traditional extraction, alkali extraction, and ultrasound assisted extraction were galactose, glucose, mannose, and arabinose. All extraction methods showed a trend of monosaccharide content with galactose greater than glucose, glucose greater than mannose, and mannose greater than arabinose. The monosaccharide content per unit mass of polysaccharide obtained by the traditional extraction method was much higher than that obtained by other extraction methods, and the alkali extraction method had the lowest content.

3. Infrared spectrum analysis showed that pine cone polysaccharide samples had characteristic absorption of intramolecular hydrogen bond $\mathrm{O}-\mathrm{H}, \mathrm{C}-\mathrm{H}$ bond, $\mathrm{C}=\mathrm{O}$ bond, $\mathrm{C}-\mathrm{O}$ bond, glycoside bond, and glucuronic acid. All samples had typical chemical structures of a polysaccharide. 
4. The polysaccharides obtained by traditional water extraction and ultrasound assisted extraction had certain scavenging effects on DPPH free radicals. Within a certain range, the scavenging effect increased with the increase of polysaccharide concentration. The DPPH scavenging rate of polysaccharides obtained by the traditional water extraction method was much higher than that obtained by ultrasound assisted extraction and the control experiment. The DPPH scavenging effect of unpurified polysaccharides was better than that of purified polysaccharides.

5. In the detection of antimicrobial activity, the ethanol extract of Pinus koraiensis raw material pretreatment has certain antimicrobial activity against E.coli.

\section{ACKNOWLEDGMENTS}

The authors are grateful for the support of the National Key R\&D Program of China (Grant No.2016YFD0600803).

\section{REFERENCES CITED}

Lin, J., Li, P., and Chen, K. S. (2013). "Advances in anti-tumor activity of polysaccharides in recent five years," China Journal of Chinese Materia Medica 38(8), 1116-1125. DOI: 10.4268/cjcmm20130802

Li, C. Z. (2013). The Isomerization of Glucose into Fructose over Sn-Beta Synthesized through Isomorphous Substitution, Master's Thesis, Dalian University of Technology, Dalian City, China.

Lv, X. M., Hu, Y. F., Liu, G. M., and Peng, F. (2010). "Research progress in chemical components and biological activities of pine cone," Medical Recapitulate 16(7), 1071-1073. DOI: 10.3969/j.issn.1006-2084.2010.07.036

Lv, Y. J., Wang, S. X., Peng, F., Li, H. Z., and Liu, G. M. (2008). "Studies on effective compositions of pine cone VI. Antitumor activity of fractions from pine cone and pine nut shell of Pinus koraiensis Sieb et Zucc and P. tabulaeformis Carr," Journal of Dali University 7(2), 1-2. DOI: 10.3969/j.issn.1672-2345.2008.02.002

Meng, Q. (2010). Separation, Purification and Bioactivity of Polysaccharide from Pine Cone of Pinus Koraiensis, Master's Thesis, Harbin Institute of Technology, Harbin City, China.

Moradali, M. F., Mostafavi, H., Ghods, S., and Hedjaroude, G.A. (2007). "Immunomodulating and anticancer agents in the realm of macromycetes fungi (macrofungi)," International Immunopharmacology 7(6), 701-724. DOI: 10.1016/j.intimp.2007.01.008

Pelissari, F. M., Grossmann, M. V. E., Fabio, Y., and Pineda, E. A. G. (2009). "Antimicrobial, mechanical, and barrier properties of cassava starch-chitosan films incorporated with oregano essential oil". Journal of Agricultural and Food Chemistry 57(16), 7499-7504. DOI: 10.1021/jf9002363

Sakagami, H., Ikeda, M., Unten, S., Takeda, K., Murayama, J., Hamada, A., Kimura, K., Komatsu, N., and Konno, K. (1987). "Antitumor activity of polysaccharide fractions from pine cone extract of Pinus parviflora Sieb.et Zucc," Anticancer Res. 7(6), 1153 1159. 
Sakagami, H., Takeda, K., Makino, Y., and Konno, K. (1986). "Partial purification of novel differentiation-inducing substances(s) from hot water extract of Japanese pine cone," Jep. J. Cancer Res. 77(1), 59-64.

Sakagami, H., Kushida, T., Oizumi, T., Nakashima, H., and Makino, T. (2010). "Distribution of lignin-carbohydrate complex in plant kingdom and its functionality as alternative medicine," Pharmacology \& Therapeutics 128(1), 91-105. DOI: 10.1016/j.pharmthera.2010.05.004.

Shen, S. Y. (2014). Study on Isolation, Purification, Structure and Antioxidant Activity of Polysaccharides from Different Parts of Pinus koraiensis, Master's Thesis, Harbin Institute of Technology, Harbin City, China.

Spizzirri, U. G., Iemma, F., Puoci, F., Cirillo, G., Curcio, M., Parisi, O. I., et al. (2009). "Synthesis of antioxidant polymers by grafting of gallic acid and catechin on gelatin," Biomacromolecules 10(7), 1923-1930. DOI: 10.1021/bm900325t

Tang, W. C., Hemm, I., and Bertram, B. (2003). "Recent development of anti-tumor agents from Chinese herbal medicines, Part II. High molecular compounds," Planta Med. 69(3), 193. DOI: 10.1055/s-2003-37718

Wang, P., Zhang, Z., and Wu, Z. J. (2015). "Study on Antioxidant experiment of Pinus koraiensis pine cone," Ginseng Research 27(3), 27-29. DOI: 10.3969/j.issn.16711521.2015.03.010

Wang, W. W., Zhang, Y. W., Long, T. R, and Wei, H. H. (2013). "Determination of polysaccharides from pine cone of Pinus koraiensis by improved-differential phenol sulfuric method," Northwest Pharmaceutical Journal 28(04), 340-342. DOI: 10.3969/j.issn.1004-2407.2013.04.005

Xiang, D., Lai, F. Y., and Liang, P. (2004). "Study on alkali extraction of pumpkin polysaccharide," Science and Technology of Food Industry 25(11), 120-122. DOI: 10.3969/j.issn.1002-0306.2004.11.038

Xu, R. J., Ye, H., Sun, Y., Tu, Y., and Zeng, X. (2012). "Preparation, preliminary characterization, antioxidant, hepatoprotective and antitumor activities of polysaccharides from the flower of tea plant (Camellia sinensis)," Food Chem Toxicol 50(7), 2473. DOI: 10.1016/j.fct.2011.10.047

Xu, R. B., Yang, X., Wang, J., Zhao, H.-T., Lu, W.-H., Cui, J., Cheng, C.-L., Zou, P., Huang, W.-W., Wang, P., Li, W.-J., and Hu, X.-L. (2012). "Chemical composition and antioxidant activities of three polysaccharide fractions from pine cones," Int. J. Mol. Sci. 13(11), 14262-14277. DOI: 10.3390/ijms131114262.

Yu, L. M., Zhao, M. M., Wang, J. S., Cui, C., Yang, B., Jiang, Y. M., and Zhao, Q. Z. (2008). "Antioxidant, immunomodulatory and anti-breast cancer activities of phenolic extract from pine (Pinus massoniana Lamb) bark," Innovative Food Science \& Emerging Technologies 9(1), 122-128. DOI: 10.1016/j.ifset.2007.06.006.

Zhang, L., Tu, Z. C., Wang, H., Wen, Q. H., Fu, Z. F., and Xie, X. (2016). “Antioxidant activity and phenolic acids profiles of Artemisia selengensis Turcz extracted with various methods by HPLC-QTOF-MS/MS," Journal of Food Biochemistry 40(4), 603-612. DOI: 10.1111/jfbc. 12255

Zhang, Y. W., and Gao, Y. (2015). "Study on antioxidant activity of polysaccharides from Pinus koraiensis pine cone residue," Chemistry \& Bioengineering 32(7), 41-43. DOI: 10.3969/j.issn.1672-5425.2015.07.011

Zhang, Z., Li, C. Y., Zheng, Y. T., and Liu, G. M. (2014). "In vitro anti-HIV activities of extracts from Pinus armandii Franch," Lishizhen Medicine and Materia Medica Research 25(1), 25-26. DOI: 10.3969/j.issn.1008-0805.2014.01.011 
Zhang, Z., Zhang, P., Han, X. D., Yang, M., Liu, F., and Liu, G. M. (2010). “Content determination of polysaccharides in the anti-HIV active sites from pine cone of Pinus armandii Franch," Lishizhen Medicine and Materia Medica Research 21(10), 24352436. DOI: 10.3969/j.issn.1008-0805.2010.10.007

Article submitted: August 22, 2019; Peer review completed: October 19, 2019; Revised version received and accepted: October 29, 2019; Published: October 31, 2019.

DOI: 10.15376/biores.14.4.9945-9956 